\title{
Fuzzy Exponential Smoothing Model and Its Application in Prediction of Unsafe Event Concerned on Aircraft Collision
}

\author{
Xu-Sheng Gan ${ }^{1, a}$, Jian-Guo Gao ${ }^{2, b}$, Huan-Bin Wang ${ }^{2, c}$ \\ ${ }^{1}$ XiJing College, Xi'an, Shaanxi, 710123, China \\ ${ }^{2}$ Material Management and Safety Engineering College, Air Force Engineering \\ University, Xi'an, Shaanxi, 710051, China \\ a ganxusheng123@163.com, b gjg123@163.com, ${ }^{\mathrm{c}}$ whb1979@163.com
}

Keywords: Exponential Smoothing; Fuzzy Theory; Unsafe Event; Aircraft Collision

\begin{abstract}
To avoid the emergence of unsafe event concerned on aircraft collision, a hybrid modeling and predicting method combined fuzzy theory and exponential smoothing method is proposed. Fuzzy theory is mainly used to solve thr difficuty in the determination of the smoothing coefficient. The validation result shows that the proposed prediction method presents a good modeling and predicting performance. It is feasible and effective to predict the unsafe event concerned on aircraft collision for management and decision-making.
\end{abstract}

\section{Introduction}

The time series analysis model [1] and Grey prediction model [2], both in parameters estimate, adopt the least square method to perform the curve fitting of historical data. It can be seen from the test result for Grey prediction model that the fitting effect between curve and historical data is very good, but the prediction is not good, because the more important in the forecast for the is fit well of the recent data, and the accurate fitting for historical data is unnecessary.

Using the exponential smoothing method in prediction of unsafe event concerned on aircraft collision, need to avoid the shortcomings for processing data in least square method. It lays particular emphasis on some dissimilarity in processing data. it is a light weight. Its dependence on recent historical data is greater, Its dependence on early historical data is smaller. The degree of dependence relies directly on the smoothing coefficient $a$, but $a$ is also a fuzzy data, the possibility of the size of its value also affects the possibility of prediction value. It is very pivotal to select the smoothing coefficient $a$ in the application of exponential smoothing principle.

According to the fuzziness between good and bad of the smoothing coefficient $a$, the basic principles of exponential smoothing method [3][4] is integrated with fuzzy mathematics theory to obtain the the optimal values of the smoothing coefficient $a$, and the actual data unsafe event concerned on aircraft collision is used to predict and analyze for method validation.

\section{Fuzzy exponential smoothing model}

In fuzzy exponential smoothing model, on the basis of exponential smoothing model, first fuzzy the smoothing exponential $a$, and determine a threshold value $\lambda(0 \leq \lambda \leq 1)$ according to the membership function of $a$, and then select $a$ that membership $\mu(x) \geq \lambda$ to get a set $A_{\lambda}$ with $\lambda$ level of fuzzy set $\stackrel{A}{\sim}$. The set is taken as the smoothing exponential set for prediction, using the exponential smoothing model to predict. we can get a group of more possible prediction value.

$$
\vec{S}_{t}^{(p)}=\left\{\begin{array}{cc}
a y_{t}+(1-a) S_{t-1}^{(p)} & p=1 \\
a S_{t}^{(p-1)}+(1-a) S_{t-1}^{(p)} & p \neq 1
\end{array}\right.
$$


where $S_{t}{ }^{(p)}$ is the $p$-order exponential smoothing value; $a$ is the smoothing exponential; $y_{t}$ is the number of unsafe event concerned on aircraft collision per year; $p$ is the exponential smoothing times; $t$ is the years.

\section{Calculation of smoothing coefficient}

To use the exponential smoothing principle, choosing a proper weight coefficient is very important, which can directly affect the prediction result.

The size of $a$ reflects the effect of data of different period in the prediction value. The greater $a$, the larger the proportion of recent data in the prediction, in the limit cases, $a=1, S_{t}^{(1)}=y_{t-1}$, i.e., the expected value at next moment is equal to the actual value at the moment; Conversely, the smaller $a$, the smaller the role of recent data, $a=0, S_{t}^{(1)}=S_{t-1}{ }^{(1)}, y_{t-1}$ does not play the role, the expected value at next moment is equal to the expected value at the moment, the actual value at the moment does not play the role.

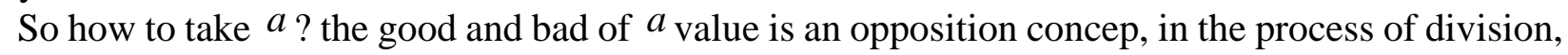
they have not the absolutely clear boundaries with intermediary transitional, is a fuzzy concept of objective existence. This is the fuzzy of optimization value and an objective attribute of things which present in the process of division of good and bad, It is also the basis of the establishment of $a_{\text {value }}$ using the fuzzy optimization theory [5].

Suppose that $X$ is the domain, namely, ${ }^{n}$ scheme of the $a_{\text {value of smoothing exponential form a }}$ solution set:

$$
X=\left\{a_{1}, a_{2}, \cdots, a_{j}, \cdots, a_{n}\right\}
$$

$a_{j}$ is the schemes or elements in the set, Then the smoothing exponential $a_{j}$ corresponds to the membership of fuzzy concept 'excellent' (denoted by $\underset{\sim}{A}): \mu_{A}\left(a_{j}\right)$; namely determine the mapping: $\mu_{A}: X \rightarrow[0,1] ; a_{j} \mid \rightarrow \mu_{A}\left(a_{j}\right)$, which the scheme with maximum membership is the optimal value of smoothing exponential $a$.

Suppose that the evaluation index set is compose of m evaluation factors or indexes for all $n$ value schemes. The evaluation of each index on $\mathrm{n}$ scheme can be expressed by index characteristic quantities, then we have the matrix of index characteristic quantities :

$$
X=\left[\begin{array}{cccc}
x_{11} & x_{12} & \cdots & x_{1 n} \\
x_{21} & x_{22} & \cdots & x_{2 n} \\
\cdots & \cdots & \cdots & \cdots \\
x_{m 1} & x_{m 2} & \cdots & x_{m n}
\end{array}\right]=\left(x_{i j}\right), \quad i=1,2, \cdots m ; j=1,2, \cdots n
$$

where $x_{i j}$ is the index characteristic quantity of $i$-th evaluation factor of $j$-th value scheme.

\section{Actual example}

To validate the proposed prediction model, we can use the actual number of unsafe event concerned on aircraft collision obtained from an air route traffic control center in China from 1985 year to 1997 year as shown in Table 1 to establish the fuzzy exponential smoothing model, and then predict the unsafe event concerned on aircraft collision from 1998 year to 2001 year by built model.

Through analysis, we can give the fitting weight of data

$$
\begin{aligned}
& w_{t}=[0.9,0.9,0.9,0.9,0.9,0.9,0.9,0.8,1,1,1,1,1] \\
& \sum_{t=1}^{13} w_{t}=12.1
\end{aligned}
$$

After normalization we can get 


$$
w_{t}=[0.074,0.074,0.074,0.074,0.074,0.074,0.074,0.066,0.083,0.083,0.083,0.083,0.083]
$$

For the calculation, $a_{\min }=0.6, a_{\max }=0.8$, the step size is 0.1 , the initial value can be obtained by calculating the average for the first four values, the results are as follows:

$$
X=[0.6,0.7,0.8]
$$

Table 1 One order smoothing value of number of unsafe event concerned on aircraft collision from 1985 year to 1997 year

\begin{tabular}{cccccc}
\hline No. & Year & Actual value & $a=0.6$ & $a=0.7$ & $a=0.8$ \\
\hline 0 & & & 76.75 & 76.75 & 76.75 \\
1 & 1985 & 92 & 85.90 & 87.43 & 88.95 \\
2 & 1986 & 76 & 79.96 & 79.43 & 78.59 \\
3 & 1987 & 65 & 70.98 & 69.33 & 67.72 \\
4 & 1988 & 74 & 72.79 & 72.60 & 72.74 \\
5 & 1989 & 49 & 58.52 & 56.08 & 53.75 \\
6 & 1990 & 33 & 43.21 & 39.92 & 37.15 \\
7 & 1991 & 24 & 31.68 & 28.78 & 26.63 \\
8 & 1992 & 20 & 24.67 & 22.63 & 21.33 \\
9 & 1993 & 22 & 23.07 & 22.19 & 21.87 \\
10 & 1994 & 21 & 21.83 & 21.36 & 21.17 \\
11 & 1995 & 16 & 18.33 & 17.61 & 17.03 \\
12 & 1996 & 12 & 14.53 & 13.68 & 13.01 \\
13 & 1997 & 10 & 11.81 & 11.10 & 10.60 \\
\hline
\end{tabular}

Table 2 Three order smoothing value of number of unsafe event concerned on aircraft collision from 1985 year to 1997 year

\begin{tabular}{cccccc}
\hline No. & Year & Actual value & $S_{t}^{(1)}$ & $S_{t}^{(2)}$ & $S_{t}^{(3)}$ \\
\hline 0 & & 76.75 & 76.95 & 77.22 \\
1 & 1985 & 92 & 88.95 & 86.55 & 84.68 \\
2 & 1986 & 76 & 78.59 & 80.18 & 81.08 \\
3 & 1987 & 65 & 67.72 & 70.21 & 72.38 \\
4 & 1988 & 74 & 72.74 & 72.23 & 72.26 \\
5 & 1989 & 49 & 53.75 & 57.45 & 60.41 \\
6 & 1990 & 33 & 37.15 & 41.21 & 45.05 \\
7 & 1991 & 24 & 26.63 & 29.55 & 32.65 \\
8 & 1992 & 20 & 21.33 & 22.97 & 24.91 \\
9 & 1993 & 22 & 21.87 & 22.09 & 22.65 \\
10 & 1994 & 21 & 21.17 & 21.35 & 21.61 \\
11 & 1995 & 16 & 17.03 & 17.89 & 18.63 \\
12 & 1996 & 12 & 13.01 & 13.99 & 14.92 \\
13 & 1997 & 10 & 10.60 & 11.28 & 12.01 \\
\hline
\end{tabular}

The matrix of index characteristic quantity is 


$$
\begin{aligned}
x & =\left[\begin{array}{lll}
0.334 & 0.231 & 0.151 \\
1.970 & 1.063 & 0.440
\end{array}\right] \\
R & =\left[\begin{array}{lll}
0 & 0.563 & 1 \\
0 & 0.593 & 1
\end{array}\right] \\
\underset{\sim}{A} & =\left[\begin{array}{lll}
0 / 0.1 & 0.85 / 0.2 & 1 / 0.3
\end{array}\right]
\end{aligned}
$$

Therefore, we can take the largest membership value $a=0.8$ as the exponential smoothing to perform the prediction calculation. Then the prediction value of three order exponential smoothing table is shown in Table 2. So we can get

$$
S_{13}^{(1)}=10.60 ; \quad S_{13}^{(2)}=11.28 ; \quad S_{13}^{(3)}=12.01
$$

The output of the establish model is

$$
\begin{aligned}
& a_{t}=9.97 ; b_{t}=1.97 ; c_{t}=0.33 \\
& Y_{t+T}=9.97-1.96 T+0.33 T^{2}
\end{aligned}
$$

The established model is used to predict the number of unsafe event concerned on aircraft collision from 1998 year to 2001 year, and compare with the actual unsafe event number, the obtained prediction results are shown in Table 3.

Table 3 Prediction results of number of unsafe event concerned on aircraft collision from 1998 year to 2001 year

\begin{tabular}{ccccc}
\hline Year & 1998 & 1999 & 2000 & 2001 \\
\hline Actual value & 9 & 7 & 7 & 6 \\
Prediction value & 8.4 & 7.5 & 6.7 & 7.1 \\
Relative error & $6.7 \%$ & $7.1 \%$ & $4.3 \%$ & $18.3 \%$ \\
\hline
\end{tabular}

The prediction error of the model is between $4.3 \% \sim 18.3 \%$, taking into account the safety practicalities of aircraft collision that occurred, this model is quite accurate in short-term prediction.

\section{Conclusions}

For the prevention of unsafe event concerned on aircraft collision, a fuzzy exponential smoothing prediction method is proposed. In the method, the fuzzy theory is introduced to determine the smoothing exponential. Actual example on unsafe event concerned on aircraft collision shows that the proposed fuzzy exponential smoothing model can give a more satisfactory prediction result with easily understanding. It is the supplement and enhancement for the existing aircraft anti-collision approach. The prediction results obtained by the model reflect the actual safety situation of aircraft anti-collision as a whole and can assist the support the management and decision-making for preventing the aircraft collision.

\section{References}

[1] G. X. Xu. Statistical forecasting and decision-making. Shanghai: Shanghai University of Finance and Economics Press, (2008)

[2] J. L. Deng. The primary methods of grey system theory. Wuhan: Huazhong University of Science and Technology Press, (1988)

[3] J. X. Xu, Y. Yan, F. H. Yan. The application of exponential smoothing model in GDP prediction of typical city. Water Conservancy Science and Technology and Economy, 14(7), (2008) , 551-554

[4] W. Shan, S. T. Zhao. Application of exponential smoothing method in the settlement forecasting of soft clay ground of highway. Forest Engineering, 23(4), (2007), 67-69

[5] Z. Z. Peng. Fuzzy mathematics and its application. Wuhan University Press, (2004) 